\title{
A note on Mersenne Padovan and Perrin numbers
}

\author{
Bir Kafle ${ }^{1}$, Salah Eddine Rihane ${ }^{2}$ and Alain Togbé $e^{3}$ \\ ${ }^{1}$ Department of Mathematics, Statistics, and Computer Science \\ Purdue University Northwest \\ 1401 S, U.S. 421, Westville IN 46391, USA \\ e-mail: bkafleapnw.edu \\ 2 Department of Mathematics and Computer Science \\ Abdelhafid Boussouf University \\ Mila 43000, Algeria \\ e-mail: salahrihane@hotmail.fr \\ ${ }^{3}$ Department of Mathematics, Statistics, and Computer Science \\ Purdue University Northwest \\ 1401 S, U.S. 421, Westville IN 46391, USA \\ e-mail: atogbe@pnw.edu
}

Received: 7 August 2020

Revised: 11 February 2021

Accepted: 1 March 2021

\begin{abstract}
In this paper, we determine all the Mersenne numbers which are in the sequences of Padovan and Perrin numbers, respectively.
\end{abstract}

Keywords: Padovan numbers, Perrin numbers, Mersenne numbers, Linear form in logarithms, Reduction method.

2010 Mathematics Subject Classification: 11B39, 11D45, $11 \mathrm{~J} 86$.

\section{Introduction}

The intersection of sequences was and continue to be an interesting subject of research. In [14], Stein examined the intersection of Fibonacci sequences. In particular, he proved that two Fibonacci sequences generally do not meet and that if they do meet at least three times, then one is simply the tail of the other. In [12] generalized Stein's work by looking at conditions for fewer than two intersections, exactly two intersections, and more than two intersections. We will continue in the same spirit by studying the intersection between the Mersenne numbers and the Padovan or Perrin numbers. 
A Mersenne number, denoted by $M_{j}$, is a number of the form

$$
M_{j}=2^{j}-1,
$$

where $j$ is a nonnegative integer. It is named after a French mathematician of the seventeenth century Marin Mersenne (1588-1648). The Mersenne sequence $\left(M_{j}\right)_{j>0}$ can be defined recursively as

$$
M_{j+2}=3 M_{j+1}-2 M_{j},
$$

with the initial values $M_{0}=0$ and $M_{1}=1$. A Mersenne prime is a Mersenne number that is prime. The first few Mersenne numbers are

$$
0,1,3,7,15,31,63,127,255,511,1023,2047,4095,8191,16383, \ldots
$$

These numbers have been studied in great depth, in particular, the Mersenne primes. An easy exercise shows that if $M_{j}$ is a prime, then $j$ is also a prime, though not all $M_{j}$ with $j$ prime are primes. In fact, given a prime number $j$, only very few numbers of the form $2^{j}-1$ are primes. For example, $M_{7}=2^{7}-1$ is a prime number, whereas $M_{11}=2047=23 \cdot 89$ is composite. The quest for Mersenne primes is still an active research field. It is conjectured that there are infinitely many Mersenne primes, [9]. The largest known Mersenne prime number, also known as $M_{82589933}$ is $2^{82,589,933}-1$, which was discovered by Patrick Laroche in December 2018. For the history, the list of known Mersenne primes and the current progress, one can see GIMPS [7]. Historically, the study of Mersenne primes was influenced by their deep connection to the perfect numbers, i.e., the numbers that are equal to the sum of their proper divisors. It is well-known that a necessary and sufficient condition that $k$ be an even perfect number is that $k=2^{j-1}\left(2^{j}-1\right)$, where $2^{j}-1$ is a Mersenne prime. The sufficiency was proved by Euclid (IV-III century BC) and the necessity was first proved two millennia late by Euler (1707-1783). However though, it is still unknown if there are any odd perfect numbers. For some of the new results, one can see $[4,6]$. A lot of interesting results concerning the Mersenne numbers can be found in the book [9], (Kř́žžek, Luca and Somer, 2001). This book also contains an extensive list of references for any interested reader.

Recently in [2], Bravo and Gómez (2016) found all $k$-Fibonacci numbers which are also Mersenne numbers. On related results, Bravo and Herrera determined all $k$-Fibonacci and $k$-Lucas numbers that are also Fermat numbers, see [3]. In [11], Rihane, Adegbindin and Togbé found all of the Padovan and Perrin numbers, which we define below, that are also in the sequence of Fermat numbers.

Let $\left(P_{m}\right)_{m \geq 0}$ be the Padovan sequence (sequence A000931 in the OEIS [13]) given by

$$
P_{m+3}=P_{m+1}+P_{m}
$$

for all $m \geq 0$, where $P_{0}=0$ and $P_{1}=P_{2}=1$. The first few terms of this sequence are

$$
\mathbf{0 , 1} \mathbf{1}, \mathbf{1}, 2,2, \mathbf{3}, 4,5, \mathbf{7}, 9,12,16,21,28,37,49,65,86,114,151,200,265,351, \cdots
$$

Similarly, let $\left(E_{m}\right)_{m \geq 0}$ be the Perrin sequence (sequence A001608 [13]) given by

$$
E_{m+3}=E_{m+1}+E_{m},
$$


for $m \geq 0$, where $E_{0}=3, E_{1}=0$ and $E_{2}=2$. The first few terms of this sequence are

$\mathbf{3 , 0}, 2, \mathbf{3}, 2,5,5, \mathbf{7}, 10,12,17,22,29,39,51,68,90,119,158,209,277,367,486, \cdots$

In the present paper, we continue this discussion and identify all Mersenne numbers in the sequences of Padovan and Perrin numbers. Our results are the following.

Theorem 1.1. The only Mersenne numbers in the Padovan sequence are $M_{0}=0, M_{1}=1$, $M_{2}=3$ and $M_{3}=7$.

Theorem 1.2. The only Mersenne numbers in the Perrin sequence are $M_{0}=0, M_{2}=3$ and $M_{3}=7$.

The outline of this paper is as follows. Our method follows similar to that of [11]. The main results, Theorems 1.1 and 1.2, come as the studies of the Diophantine equations

$$
P_{n}=2^{m}-1
$$

and

$$
E_{n}=2^{m}-1
$$

in nonnegative integers $(n, m)$, respectively. For this, in Sections 2 and 3, we state some of the results that are useful in studying the equations (1) and (2). Particularly, we recall some of the properties of Padovan and Perrin numbers (Section 2), a result of Matveev [10] that we will use to obtain the lower bounds for linear forms in logarithms of algebraic numbers, de Weger reduction method, [16]. In the last two sections, we will completely prove our results using Baker method and the reduction method (subsection 3.2).

\section{Auxiliary results}

First, we recall some facts and properties of the Padovan and the Perrin sequences $\left(P_{n}\right)_{n \geq 0}$ and $\left(E_{n}\right)_{n \geq 0}$, respectively, which will be used later. One can also see [11]. The characteristic equation

$$
x^{3}-x-1=0
$$

has roots $\alpha, \beta, \gamma=\bar{\beta}$, where

$$
\alpha=\frac{\omega_{1}+\omega_{2}}{6}, \quad \beta=\frac{-\omega_{1}-\omega_{2}+i \sqrt{3}\left(\omega_{1}-\omega_{2}\right)}{12},
$$

and

$$
\omega_{1}=\sqrt[3]{108+12 \sqrt{69}}, \quad \omega_{2}=\sqrt[3]{108-12 \sqrt{69}}
$$

Let

$$
\begin{aligned}
& c_{\alpha}=\frac{(1-\beta)(1-\gamma)}{(\alpha-\beta)(\alpha-\gamma)}=\frac{1+\alpha}{-\alpha^{2}+3 \alpha+1}, \\
& c_{\beta}=\frac{(1-\alpha)(1-\gamma)}{(\beta-\alpha)(\beta-\gamma)}=\frac{1+\beta}{-\beta^{2}+3 \beta+1}, \\
& c_{\gamma}=\frac{(1-\alpha)(1-\beta)}{(\gamma-\alpha)(\gamma-\beta)}=\frac{1+\gamma}{-\gamma^{2}+3 \gamma+1}=\overline{c_{\beta}} .
\end{aligned}
$$


With these notations, Binet's formula for $P_{n}$ is given by

$$
P_{n}=c_{\alpha} \alpha^{n}+c_{\beta} \beta^{n}+c_{\gamma} \gamma^{n} \text { for all } n \geq 0,
$$

and Binet's formula for $E_{n}$ is given by

$$
E_{n}=\alpha^{n}+\beta^{n}+\gamma^{n}, \text { for all } n \geq 0 .
$$

Numerically, we have

$$
\begin{aligned}
& 1.32<\alpha<1.33 \\
& 0.86<|\beta|=|\gamma|=\alpha^{-1 / 2}<0.87 \\
& 0.72<c_{\alpha}<0.73 \\
& 0.24<\left|c_{\beta}\right|=\left|c_{\gamma}\right|<0.25
\end{aligned}
$$

Furthermore, by induction on $n$, we can prove that

$$
\alpha^{n-2} \leq P_{n} \leq \alpha^{n-1}, \quad \text { for all } n \geq 4
$$

and

$$
\alpha^{n-2} \leq E_{n} \leq \alpha^{n+1}, \quad \text { for all } n \geq 2
$$

\section{The tools}

The next tools are related to the transcendental approach to solve Diophantine equations. For any non-zero algebraic number $\gamma$ of degree $d$ over $\mathbb{Q}$, whose minimal polynomial over $\mathbb{Z}$ is $a \prod_{j=1}^{d}\left(X-\gamma^{(j)}\right)$, we denote by

$$
h(\gamma)=\frac{1}{d}\left(\log |a|+\sum_{j=1}^{d} \log \max \left(1,\left|\gamma^{(j)}\right|\right)\right)
$$

the usual absolute logarithmic height of $\gamma$. We also require some of the properties of the absolute logarithmic height of algebraic numbers. These properties are contained in Lemma 3.1 below.

Lemma 3.1. [15, Property 3.3, Page 75] For algebraic numbers $\gamma$ and $\eta$, we have

$$
h(\gamma \cdot \eta) \leq h(\gamma)+h(\eta)
$$

and

$$
h(\gamma+\eta) \leq h(\gamma)+h(\eta)+\log 2 .
$$

Moreover, for any algebraic number $\zeta \neq 0$ and for any $m \in \mathbb{Z}$, we have

$$
h\left(\zeta^{m}\right)=|m| h(\zeta) .
$$

\subsection{Linear forms in logarithms}

To prove Theorems 1.1 and 1.2, we use lower bounds for linear forms in logarithms to bound the index $n$ appearing in equations (1) and (2). We need the following general lower bound for linear forms in logarithms due to Matveev [10]. 
Theorem 3.2. Let $\gamma_{1}, \ldots, \gamma_{\ell}$ be real algebraic numbers and let $b_{1}, \ldots, b_{\ell}$ be nonzero rational integer numbers. Let $D$ be the degree of the number field $\mathbb{Q}\left(\gamma_{1}, \ldots, \gamma_{\ell}\right)$ over $\mathbb{Q}$ and let $A_{j}$ be a positive real number satisfying

$$
A_{j}=\max \{D h(\gamma),|\log \gamma|, 0.16\} \text { for } j=1, \ldots, \ell .
$$

Assume that

$$
B \geq \max \left\{\left|b_{1}\right|, \ldots,\left|b_{\ell}\right|\right\}
$$

If $\gamma_{1}^{b_{1}} \cdots \gamma_{\ell}^{b_{\ell}}-1 \neq 0$, then

$$
\left|\gamma_{1}^{b_{1}} \cdots \gamma_{\ell}^{b_{\ell}}-1\right| \geq \exp \left(-1.4 \cdot 30^{\ell+3} \cdot \ell^{4.5} \cdot D^{2}(1+\log D)(1+\log B) A_{1} \cdots A_{\ell}\right) .
$$

\subsection{De Weger reduction method}

The upper bound of $n$ we obtain so far is generally too large. Therefore, the next step is to reduce it further to a reasonable level. For this reduction purpose, we present a variant of the reduction method of Baker and Davenport due to de Weger [16].

Let $\vartheta_{1}, \vartheta_{2}, \beta \in \mathbb{R}$ be given, and let $x_{1}, x_{2} \in \mathbb{Z}$ be unknowns. Let

$$
\Lambda=\beta+x_{1} \vartheta_{1}+x_{2} \vartheta_{2} .
$$

Let $c, \delta$ be positive constants. Set $X=\max \left\{\left|x_{1}\right|,\left|x_{2}\right|\right\}$. Let $X_{0}, Y$ be positive. Assume that

$$
\begin{gathered}
|\Lambda|<c \cdot \exp (-\delta \cdot Y), \\
Y \leq X \leq X_{0} .
\end{gathered}
$$

When $\beta=0$ in (9), we get

$$
\Lambda=x_{1} \vartheta_{1}+x_{2} \vartheta_{2} .
$$

Put $\vartheta=-\vartheta_{1} / \vartheta_{2}$. We assume that $x_{1}$ and $x_{2}$ are coprime. Let the continued fraction expansion of $\vartheta$ be given by

$$
\left[a_{0}, a_{1}, a_{2}, \ldots\right]
$$

and let the $k$-th convergent of $\vartheta$ be $p_{k} / q_{k}$ for $k=0,1,2, \ldots$. We may assume without loss of generality that $\left|\vartheta_{1}\right|<\left|\vartheta_{2}\right|$ and that $x_{1}>0$. We have the following results.

Lemma 3.3. [16, Lemma 3.2] Let

$$
A=\max _{0 \leq k \leq Y_{0}} a_{k+1}
$$

where

$$
Y_{0}=-1+\frac{\log \left(\sqrt{5} X_{0}+1\right)}{\log \left(\frac{1+\sqrt{5}}{2}\right)} .
$$

If (10) and (11) hold for $x_{1}, x_{2}$ and $\beta=0$, then

$$
Y<\frac{1}{\delta} \log \left(\frac{c(A+2) X_{0}}{\left|\vartheta_{2}\right|}\right)
$$

When $\beta \neq 0$ in (9), put $\vartheta=-\vartheta_{1} / \vartheta_{2}$ and $\psi=\beta / \vartheta_{2}$. Then we have

$$
\frac{\Lambda}{\vartheta_{2}}=\psi-x_{1} \vartheta+x_{2} \text {. }
$$


Let $p / q$ be a convergent of $\vartheta$ with $q>X_{0}$. For a real number $x$, we let $\|x\|=\min \{|x-n|, n \in$ $\mathbb{Z}\}$ be the distance from $x$ to the nearest integer. We have the following result.

Lemma 3.4. [16, Lemma 3.3] Suppose that

$$
\|q \psi\|>\frac{2 X_{0}}{q}
$$

Then, the solutions of (10) and (11) satisfy

$$
Y<\frac{1}{\delta} \log \left(\frac{q^{2} c}{\left|\vartheta_{2}\right| X_{0}}\right)
$$

\section{Proof of Theorem 1.1}

First, we explore a relation between $n$ and $m$. By combining equation (1) with the inequality (7), we get

$$
\alpha^{n-2} \leq P_{n}=2^{m}-1<2^{m}
$$

and

$$
2^{m-1}<2^{m}-1=P_{n} \leq \alpha^{n-1},
$$

which hold for all $n \geq 5$ and $m \geq 1$. In the case when $n<4$ and $m<1$, the only solution to the equation (1) is $(0,0)$. Now, taking logarithms on both sides of the inequalities (13) and (14) and putting them together, we obtain

$$
(n-2) \frac{\log \alpha}{\log 2}<m<(n-1) \frac{\log \alpha}{\log 2}+1 .
$$

To make our computation simpler, let us first assume that $n \leq 200$. Then, the inequality (15) above implies that $m \leq 83$. A simple computation in Maple reveals that the only solution of the equation (1) in this range are

$$
(n, m) \in\{(0,0),(1,1),(2,1),(3,1)(6,2),(9,3)\} .
$$

This means that $0,1,3$ and 7 are the only Mersenne numbers that are also Padovan numbers as listed in Theorem 1.1 in this range.

Next, we consider the situation when $n>200$. Then the inequality (15) implies, in general, that

$$
m \leq n
$$

Therefore, to solve equation (1), it is sufficient to solve it for a good upper bound for $n$. The rest of our work focuses on it.

Next, we rewrite equation (1) using the Binet's formula (4) and taking the absolute values as

$$
\left|2^{m}-c_{\alpha} \alpha^{n}\right| \leq\left|c_{\beta} \beta^{n}+c_{\gamma} \gamma^{n}+1\right|<1.44 .
$$

Dividing both sides of the last inequality by $c_{\alpha} \alpha^{n}$, we get

Let us put

$$
\left|2^{m} c_{\alpha}^{-1} \alpha^{-n}-1\right|<\frac{2}{\alpha^{n}} .
$$

$$
\Gamma_{1}:=2^{m} c_{\alpha}^{-1} \alpha^{-n}-1
$$


Now, we apply Matveev's result Theorem 3.2 on $\Gamma_{1}$. First, we need to determine that it is nonzero. If it were indeed zero, then we would get $2^{m}=c_{\alpha} \alpha^{n}$. But this is not true since if we conjugate this relation by the automorphism of Galois $\sigma:=(\alpha \beta)$ and taking the absolute values, we would get $1<2^{m}=\left|c_{\beta}\right| \cdot\left|\beta^{n}\right|<1$, which is false. In order to apply Theorem 3.2, we take $\ell:=3$ and

$$
\gamma_{1}:=2, \quad \gamma_{2}:=c_{\alpha}, \quad \gamma_{3}:=\alpha
$$

and

$$
b_{1}:=m, \quad b_{2}:=-1, \quad b_{3}:=-n .
$$

The algebraic numbers $\gamma_{1}, \gamma_{2}$ and $\gamma_{3}$ all belong to $\mathbb{Q}(\alpha)$, and so we take $D=3$. Also, $B=\max \{1, m, n\}$. Furthermore, $h\left(\gamma_{1}\right)=\log 2$ and $h\left(\gamma_{3}\right)=\frac{\log \alpha}{3}$. Further, the minimal polynomial of $c_{\alpha}$ is $23 x^{3}-23 x^{2}+6 x-1$, which has roots $c_{\alpha}, c_{\beta}, c_{\gamma}$. Also, $\max \left\{\left|c_{\alpha}\right|,\left|c_{\beta}\right|,\left|c_{\gamma}\right|\right\}<1$ (see (6)). Thus, $h\left(\gamma_{2}\right)=(\log 23) / 3$. Hence, we can take

$$
B:=n, \quad A_{1}:=2.1, \quad A_{2}:=3.14, \quad A_{3}:=\log \alpha .
$$

Now, by Theorem 3.2 and with a straightforward calculation, we obtain the following estimate:

$$
\begin{aligned}
\left|\Gamma_{1}\right| & \geq \exp \left(-1.79 \cdot 10^{13} \cdot \log \alpha \cdot(1+\log n)\right) \\
& >\exp \left(-3.57 \cdot 10^{13} \cdot \log \alpha \cdot \log n\right),
\end{aligned}
$$

where we also used the fact that $1+\log n<2 \log n$. Comparing the inequalities (17) and (18), and solving for $n$, we obtain

$$
n<1.25 \cdot 10^{15} \text {. }
$$

As we see, the bound we obtained for $n$ is very large. So, our next step is to reduce it to a reasonable value. For this purpose, we use Lemma 3.4. Recall that

$$
\Gamma_{1}:=2^{m} c_{\alpha}^{-1} \alpha^{-n}-1=e^{\Lambda_{1}}-1,
$$

where we set

$$
\Lambda_{1}:=m \log 2-n \log \alpha+\log \left(1 / c_{\alpha}\right) .
$$

Since $\Gamma_{1} \neq 0$, it follows that $\Lambda_{1} \neq 0$. So, we have two possibilities. If $\Lambda_{1}>0$, then we get

$$
0<\Lambda_{1}<\left|e^{\Lambda_{1}}-1\right|=\left|\Gamma_{1}\right|<\frac{2}{\alpha^{n}} .
$$

If $\Lambda_{1}<0$, then $1-e^{\Lambda_{1}}=\left|\Gamma_{1}\right|<\frac{1}{2}$, as $n>200$. Therefore, we have

$$
0<\left|\Lambda_{1}\right|<e^{\left|\Lambda_{1}\right|}-1=e^{\left|\Lambda_{1}\right|}\left|\Gamma_{1}\right|<\frac{4}{\alpha^{n}} .
$$

Combining both of these cases, we have

$$
0<\left|\log \left(1 / c_{\alpha}\right)+n(-\log \alpha)+m \log 2\right|<4 \exp (-n \log \alpha) .
$$

Now, we are in a situation to apply Lemma 3.4 as we planned. For this, we can take $X_{0}:=1.25 \cdot 10^{15}$. Further, we choose

$$
\begin{gathered}
c:=4, \quad \delta:=\log \alpha, \quad \beta:=\log \left(1 / c_{\alpha}\right) \\
\left(\vartheta_{1}, \vartheta_{2}\right):=(-\log \alpha, \log 2), \quad \vartheta:=\frac{\log \alpha}{\log 2}, \quad \psi:=\frac{\log \left(1 / c_{\alpha}\right)}{\log 2} .
\end{gathered}
$$


Using Maple, we find that $q_{41}=22636316802853379$ satisfies the hypotheses of Lemma 3.4. Furthermore, Lemma 3.4 implies that

$$
n<\frac{1}{\delta} \cdot \log \left(\frac{22636316802853379^{2} \cdot 4}{\log 2 \cdot 1.25 \cdot 10^{15}}\right)<200,
$$

which contradicts our assumption that $n>200$. This proves Theorem 1.1.

\section{Proof of Theorem 1.2}

In this section, we will prove Theorem 1.2, using the above method in the proof of Theorem 1.1. For the sake of completeness, we will give almost all of the details.

First, we rewrite equation (2) using the inequality (8) to obtain the following useful inequalities:

$$
\alpha^{n-2} \leq E_{n}=2^{m}-1<2^{m}
$$

and

$$
2^{m-1}<2^{m}-1=E_{n} \leq \alpha^{n+1},
$$

which hold for all $n \geq 2$ and $m \geq 1$. In the case when $n<2$ and $m<1$, the only solution of the equation (2) is $(1,0)$. Now, taking logarithms on both sides of the inequalities (20) and (21) and combining them, we obtain

$$
(n-2) \frac{\log \alpha}{\log 2}<m<(n+1) \frac{\log \alpha}{\log 2}+1 .
$$

As before, we first assume that $n \leq 125$. So, by inequality (22), we have $m \leq 52$. In this range, a quick computation in Maple reveals that the only solutions of the equation (2) in nonnegative integers $(n, m)$ are

$$
(n, m) \in\{(0,2),(1,0),(3,2),(7,3)\} .
$$

That is, the only Mersenne numbers in the Perrin sequence are 0,3 , and 7 , which are listed in the Theorem 1.2 in this range.

From now on, we assume that $n>125$. Then, the inequality also tells us that

$$
m<n \text {. }
$$

Therefore, to completely solve equation (2), we would try to obtain a reasonable upper bound for $n$. As before, using the Binet's formula (5), we rewrite equation (2) as

$$
2^{m}-\alpha^{n}=\beta^{n}+\gamma^{n}+1
$$

from which we can deduce that

$$
\left|2^{m}-\alpha^{n}\right|<2.74
$$

Dividing both sides of the last inequality by $\alpha^{n}$, we get

$$
\left|2^{m} \alpha^{-n}-1\right|<\frac{2.74}{\alpha^{n}} .
$$

Let us put

$$
\Gamma_{2}:=2^{m} \alpha^{-n}-1 .
$$


Now, we plan to apply Matveev's result Theorem 3.2 on $\Gamma_{2}$. Similarly as in the case of $\Gamma_{1}$ above, we have $\Gamma_{2} \neq 0$. In order to apply Theorem 3.2 , we take $\ell:=2$ and

$$
\gamma_{1}:=2, \quad \gamma_{2}:=\alpha, \quad b_{1}:=m, \quad b_{2}:=-n .
$$

The algebraic numbers $\gamma_{1}$ and $\gamma_{2}$ both belong to $\mathbb{Q}(\alpha)$, so we take $D=3$. Also, $B=\max \{1, m, n\}$. As before, we choose

$$
B:=n, \quad A_{1}:=2.1, \quad A_{2}:=\log \alpha .
$$

Now, Theorem 3.2 gives us the following estimate:

$$
\begin{aligned}
\left|\Gamma_{2}\right| & \geq \exp \left(-1.4 \cdot 30^{5} \cdot 2^{4.5} \cdot 3^{2} \cdot(1+\log 3) \cdot(1+\log n) \cdot 2.1 \cdot \log \alpha\right) \\
& >\exp \left(-6.11 \cdot 10^{10} \cdot \log \alpha \cdot \log n\right),
\end{aligned}
$$

where we also used the fact that $1+\log n<2 \log n$. Comparing the inequalities (24) and (25), and solving for $n$, we obtain

$$
n<1.77 \cdot 10^{12} \text {. }
$$

Next, we attempt to reduce the upper bound for $n$ further by using Lemma 3.3. Recall that

$$
\Gamma_{2}:=2^{m} \alpha^{-n}-1=e^{\Lambda_{2}}-1,
$$

where we set

$$
\Lambda_{2}:=m \log 2-n \log \alpha .
$$

Since $\Gamma_{2} \neq 0$, which follows that $\Lambda_{2} \neq 0$. If $\Lambda_{2}>0$, then we get

$$
0<\Lambda_{2}<\left|e^{\Lambda_{2}}-1\right|=\left|\Gamma_{2}\right|<\frac{2.74}{\alpha^{n}} .
$$

If $\Lambda_{2}<0$, then $1-e^{\Lambda_{2}}=\left|\Gamma_{2}\right|=\left|\Gamma_{2}\right|<\frac{1}{2}$, as $n>125$. Therefore, we have

$$
0<\left|\Lambda_{2}\right|<e^{\left|\Lambda_{2}\right|}-1=e^{\left|\Lambda_{2}\right|}\left|\Gamma_{2}\right|<\frac{5.48}{\alpha^{n}} .
$$

Combining both of these cases, we have

$$
0<|n(-\log \alpha)+m \log 2|<5.48 \exp (-n \log \alpha) .
$$

As planned, to apply Lemma 3.3 we take $X_{0}:=1.77 \cdot 10^{12}$ (by (26)), which also gives us $Y_{0}=59.2784846677 \ldots$. We furthermore choose

$$
c:=5.48, \quad \delta:=\log \alpha, \quad\left(\vartheta_{1}, \vartheta_{2}\right):=(-\log \alpha, \log 2), \quad \vartheta:=\frac{\log \alpha}{\log 2} .
$$

Using Maple, we find that

$$
A:=\max _{0 \leq k \leq Y_{0}} a_{k+1}=80 .
$$

Finally, by Lemma 3.3 we find that

$$
n<\frac{1}{\log \alpha} \cdot \log \left(\frac{4.96 \cdot(80+2) \cdot 1.77 \cdot 10^{12}}{\log 2}\right) \leq 124,
$$

which contradicts our assumption that $n>125$. This completes the proof of Theorem 1.2.

\section{Acknowledgements}

B. K and A. T. are partially supported by Purdue University Northwest, IN. 


\section{References}

[1] Baker, A., \& Davenport, H. (1969). The equations $3 x^{2}-2=y^{2}$ and $8 x^{2}-7=z^{2}$. The Quarterly Journal of Mathematics, Oxford, Ser. (2), 20, 129-137.

[2] Bravo, J. J., \& Gómez, C. A. (2020). Mersenne k-Fibonacci numbers. Glasnik Matematički, 71, 307-319.

[3] Bravo, J. J., \& Herrera, J. L. (2020). Fermat $k$-Fibonacci and $k$-Lucas numbers. Mathematica Bohemica, 145(1), 19-32.

[4] Catarino, P., Campos, H., \& Vasco, P. (2016). On the Mersenne sequence. Annales Mathematicae et Informaticae, 46, 37-53.

[5] Dujella, A., \& Pethô, A. (1998). A generalization of a theorem of Baker and Davenport. The Quarterly Journal of Mathematics, Oxford, Ser. (2), 49(195), 291-306.

[6] Goy, T. (2018). On new identities for Mersenne numbers. Applied Mathematics E-Notes, 18, $100-105$.

[7] Great Internet Mersenne Prime Search (GIMPS), Finding world record primes since 1996, published electronically at https: //www. mersenne.org/.

[8] Gúzman, S. S., \& Luca, F. (2014). Linear combinations of factorials and $S$-units in a binary recurrence sequences. Annales mathématiques du Québec, 38, 169-188.

[9] Kř́ížek, M., Luca, F., \& Somer, L. (2001). 17 Lectures on Fermat Numbers: From Number Theory to Geometry, CMS Books in Mathematics, Springer-Verlag, New York.

[10] Matveev, E. M. (2000). An explicit lower bound for a homogeneous rational linear form in the logarithms of algebraic numbers, II. Izvestiya: Mathematics, 64(6), 1217-1269.

[11] Rihane, S. E., Adegbindin, C. A., \& Togbé, A. (2020). Fermat Padovan and Perrin numbers. Journal of Integer Sequences, 23(6), Article 20.6.2.

[12] Shannon, A. G. (1983). Intersections of second order linear recursive sequences. The Fibonacci Quarterly, 21, 6-12.

[13] Sloane, N. J. A. The Online Encyclopedia of Integer Sequences, published electronically at https://oeis.org/.

[14] Stein, S. K. (1962). The intersection of Fibonacci sequences. Michigan Mathematics Journal, 9, 399-402.

[15] Waldshmidt, M. (2000). Diophantine Approximation on Linear Algebraic Groups: Transcendence Properties of the Exponential Function in Several Variables, Springer-Verlag, Berlin Heidelberg.

[16] De Weger, B. M. M. (1987). Algorithms for Diophantine Equations, $\mathrm{PhD}$ thesis, University of Leiden. 